\title{
Adenoviral transfer of HSP-70 into pulmonary epithelium ameliorates experimental acute respiratory distress syndrome
}

\author{
Yoram G. Weiss, ${ }^{1,2}$ Alina Maloyan, ${ }^{2}$ John Tazelaar, ${ }^{3}$ Nichelle Raj, ${ }^{1}$ \\ and Clifford S. Deutschman ${ }^{1}$ \\ ${ }^{1}$ Department of Anesthesia, University of Pennsylvania, Philadelphia, Pennsylvania, USA \\ ${ }^{2}$ Department of Anesthesia and Critical Care Medicine, Hadassah Medical Center, Hebrew University, Jerusalem, Israel \\ ${ }^{3}$ Department of Pathology, University of Pennsylvania, Philadelphia, Pennsylvania, USA
}

The acute respiratory distress syndrome (ARDS) provokes three pathologic processes: unchecked inflammation, interstitial/alveolar protein accumulation, and destruction of pulmonary epithelial cells. The highly conserved heat shock protein HSP-70 can limit all three responses but is not appropriately expressed in the lungs after cecal ligation and double puncture (2CLP), a clinically relevant model of ARDS. We hypothesize that restoring expression of HSP-70 using adenovirus-mediated gene therapy will limit pulmonary pathology following 2 CLP. We administered a vector containing the porcine HSP-70 cDNA driven by a CMV promoter (AdHSP) into the lungs of rats subjected to 2CLP or sham operation. Administration of AdHSP after either sham operation or 2CLP increased HSP-70 protein expression in lung tissue, as determined by immunohistochemistry and Western blot hybridization. Administration of AdHSP significantly attenuated interstitial and alveolar edema and protein exudation and dramatically decreased neutrophil accumulation, relative to a control adenovirus. CLP-associated mortality at 48 hours was reduced by half. Modulation of HSP-70 production reduces pathologic changes and may improve outcome in experimental ARDS.

J. Clin. Invest. 110:801-806 (2002). doi:10.1172/JCI200215888.

\section{Introduction}

Sepsis and the related systemic inflammatory response and multiple organ dysfunction syndromes are the leading causes of death in patients in surgical intensive care units $(1,2)$. The lung is the organ most often affected in multiple organ dysfunction syndrome, with pulmonary dysfunction taking the form of acute respiratory distress syndrome (ARDS). While true epidemiological data are scarce, Ware and Matthay estimate an incidence of 75 cases $/ 100,000$ population. This results in more than 200,000 cases/year in the USA (3). Recent data indicate that mortality from ARDS is $31 \%(2-4)$. Thus, there are approximately 80,000 deaths from ARDS yearly in the USA. The number worldwide may exceed half a million.

By and large, treatment of ARDS is supportive because the pathophysiology of this highly lethal disease is poorly understood. However, ARDS is known to

Received for publication May 8, 2002, and accepted in revised form July 9, 2002.

Address correspondence to: Clifford S. Deutschman, Department of Anesthesia, Dulles 773/Hospital of the University of Pennsylvania, 3400 Spruce Street, Philadelphia, Pennsylvania 19104-4283, USA. Phone: (215) 662-3758; Fax: (215) 349-5078; E-mail: deutschcl@uphs.upenn.edu.

Conflict of interest: No conflict of interest has been declared.

Nonstandard abbreviations used: acute respiratory distress syndrome (ARDS); cecal ligation and double puncture (2CLP); heat shock protein 70 (HSP-70); green fluorescent protein (GFP). involve unchecked inflammation. This is manifest by an influx of polymorphonuclear leukocytes, massive interstitial and alveolar accumulation of denatured protein and fluid, and a reduction in essential products, such as surfactant and surfactant proteins synthesized by pneumocytes, especially type II alveolar epithelial cells. Ultimately, the process damages and even destroys type I and type II alveolar epithelial cells (3). An intervention that limits inflammation and formation of proteo-rich edema fluid, increases synthetic capacity, and ultimately prevents cell loss would be of benefit in both treating ARDS and investigating the mechanisms that lead to its development.

Our previous studies in cecal ligation and double puncture (2CLP), looking at liver and lung, have demonstrated an alteration in protein expression that suggests a potentially useful therapeutic approach (5-9). Expression of molecules that increase neutrophil accumulation, such as the chemokine CINC (10) or a number of vascular and epithelial adhesion molecules (11), are increased after cecal ligation and puncture. On the other hand, the transcription and accumulation of key proteins that are essential for normal organ function are decreased (6-9). Importantly, several recent studies indicate that 2CLP, sepsis, and endotoxemia impair the expression of heat shock protein 70 (HSP-70) (5, 12-14). Data show that HSP-70 can limit inflammatory responses, protect proteins from damage, restore 
function to proteins that are damaged, and prevent cellular destruction (15-30). Each of these processes is a key component of ARDS pathophysiology. The deficit in HSP-70 expression represents a key failure of endogenous protective systems that might be of importance in the development of ARDS. Furthermore, restitution of an appropriate HSP-70 response might be protective. The impact of the inappropriate loss of HSP-70 expression is the focus of this study. Specifically, we test the hypothesis that use of an adenoviral vector to correct the relative defect in HSP-70 expression will prevent neutrophil accumulation, reduce protein-rich edema fluid, and improve the outcome in ARDS secondary to 2CLP.

\section{Methods}

Induction of sepsis. All animal studies were approved by the University Laboratory Animal Resources committees in both collaborating institutions and conformed to NIH standards. As previously described, severe sepsis was induced in male Sprague-Dawley rats (Charles River Laboratories, Boston, Massachusetts, USA) weighing 250-275 g under isoflurane anesthesia using 2CLP with an 18-gauge needle (5, 12). Sham-operated and unoperated animals served as controls. At the time of operation, tracheal cannulation was used to administer PBS alone or an adenovirus expressing either HSP-70 (AdHSP) or green fluorescent protein (AdGFP) (12). After the procedure, animals were fluid resuscitated with $40 \mathrm{ml} / \mathrm{kg}$ sterile saline injected subcutaneously, awakened, and allowed free access to water and food. Fluid resuscitation was repeated 24 hours after the procedure. At 48 hours after surgery, rats were reanesthetized with $50 \mathrm{mg} / \mathrm{kg}$ intraperitoneal pentobarbital and then sacrificed by exsanguination. One lung was fixed for staining and the other was homogenized and protein was isolated (14). Separate groups of sham-operated animals were administered virus and sacrificed 1, 2, 3 , or 4 weeks later.

Virus administration. Recombinant E1,E3-deleted adenoviruses expressing AdGFP or AdHSP driven by a cytomegalovirus promoter were constructed by the vector core of the Institute for Human Gene Therapy at the University of Pennsylvania School of Medicine. Vector was resuspended in PBS within the 1 hour prior to administration to avoid a decline in viral titer. A total dose of $10^{11}$ viral plaque-forming units in PBS (total volume, $300 \mu \mathrm{l}$ ) was delivered in three aliquots. Animals in these cohorts were also subjected to 2CLP or sham operation.

Protein immunoblotting. Immunoblotting was performed on $10-\mu \mathrm{g}$ samples as previously described (14). Blots were labeled with a primary polyclonal goat antibody to rat HSP-70 (Santa Cruz Biotechnology Inc., Santa Cruz, California, USA) and secondarily exposed to rabbit anti-goat IgG (Santa Cruz Biotechnology Inc.). The signal was detected with enhanced chemiluminescence (ECL; Amersham Pharmacia Biotech, Piscataway, New Jersey, USA), and density was measured using scanning densitometry.

Immunobistochemical quantification of HSP-70 in lung parenchyma. Immunostaining was performed as previously described $(5,14)$. The primary antibody was a 1:500 dilution of goat polyclonal anti-rat HSP-70 (Santa Cruz Biotechnology Inc.). A secondary anti-goat IgG (Santa Cruz Biotechnology Inc.) conjugated to horseradish peroxidase was added, and detection was performed using immunoperoxidase/avidin/biotin methodology (TSA-Indirect; PerkinElmer Life Sciences Inc., Boston, Massachusetts, USA). Metal-enhanced 3,3-diaminobenzidine (Pierce Biotechnology Inc., Rockford, Illinois, USA) was added, and after 5 minutes, sections were washed, treated briefly with ethanol and xylene, dried, and mounted.

Histology and neutrophil accumulation in lung parenchyma. Sections from each animal were stained with hematoxylin and eosin. Ten high-powered fields were selected at random from each representative section, and neutrophils were identified and counted by a pathologist (J. Tazelaar). Neutrophils per high-powered field for each animal were averaged, and mean and SD for all animals in a given cohort were calculated.

Statistical analysis. ANOVA with Bonferroni correction was used to examine differences between and within groups. Significance level was set at $P<0.05$.

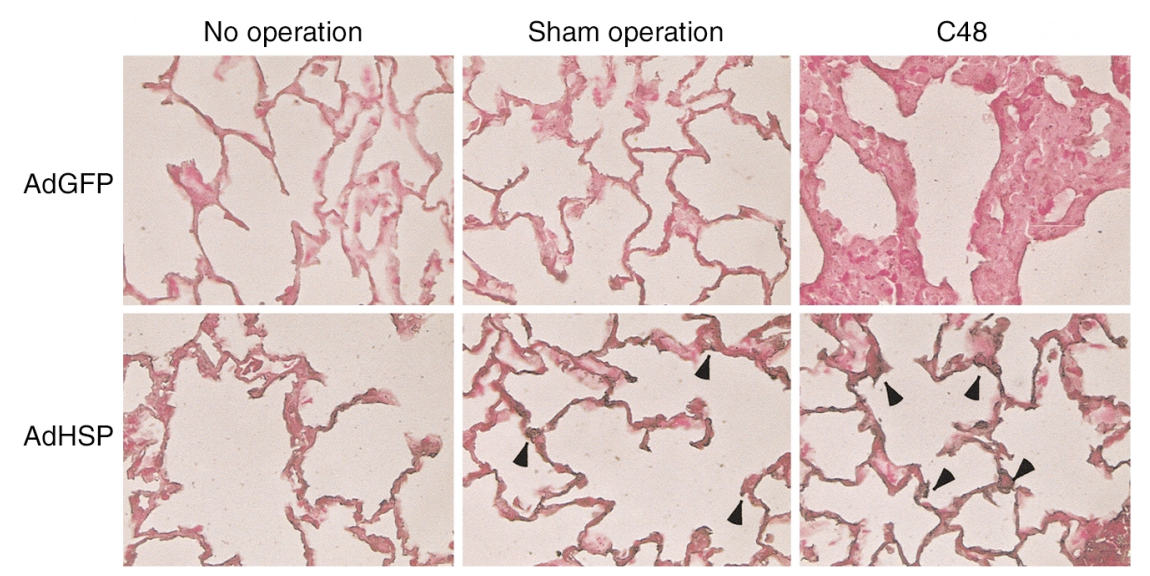

\section{Figure 1}

Representative HSP-70-immunostained lung sections obtained following administration of AdGFP or AdHSP. C48 indicates 2CLP with virus administration; tissue was fixed 48 hours afterward. Primary antibody was polyclonal goat anti-rat HSP-70; secondary antibody was rabbit antigoat IgG conjugated to horseradish peroxidase. Detection was performed with immunoperoxidase/avidin/biotin and metal-enhanced 3,3-diamino-benzidine. Arrows indicate HSP-70 staining, primarily in type II pulmonary epithelial cells. 


\section{Results}

AdHSP administration increases HSP-70 expression in the lung. To determine the extent to which AdHSP expression increased expression of HSP-70 by pulmonary epithelial cells after 2CLP or sham operation, we performed immunostaining and Western blot hybridization. Figure 1 depicts representative HSP-70-stained sections obtained from unoperated animals, shamoperated animals, or 2CLP animals after administration of AdHSP or AdGFP. This demonstrates immunohistochemical evidence of enhanced HSP-70 protein expression in 2CLP animals 48 hours after AdHSP treatment. Expression in unoperated animals was minimal. Previous studies have indicated low levels of viral uptake in the absence of operative intervention (14). Uptake was primarily in type II pulmonary epithelial cells, consistent with previous findings (14).

Immunoblotting of lung homogenate further substantiates increased HSP-70 expression. Representative immunoblots are shown in Figure 2a. Quantitative data was derived from autoradiograms using laser densitometry (Figure 2b). These data demonstrate that, relative to animals administered no virus, AdHSP significantly increased levels of HSP-70 in unoperated animals, sham-operated animals, and animals subjected to 2CLP. To demonstrate that increases in HSP-70 expression did not occur as a result of a nonspecific response to virus administration, a group of 2CLP animals were given AdGFP (data not shown). No increase in HSP-70 expression was detected in these animals. This is consistent with immunostaining results depicted in Figure 1.

Time course of AdGFP or AdHSP expression in lung tissue following sham operation. As demonstrated in Figure 2, a and $b$, expression of HSP-70 was increased 48 hours following 2CLP and AdHSP administration. However, adenoviral vector administration has been associated with the development of lymphocytic inflammatory infiltrate $(11,31,32)$, a particular concern if expression is persistent $(30,31)$. Therefore, we used immunoblotting to examine the time course of HSP-70 expression following sham operation and AdHSP administration (Figure 2c, Table 1). HSP-70 expression was increased 48 hours after AdHSP inoculation. However, by 1 week after virus administration/sham operation, expression had returned to baseline levels. Analyses performed up to 4 weeks following AdHSP administration indicated no increase in HSP-70 expression. Importantly, no lymphocytic infiltrate was detected.

AdHSP attenuates lung injury following 2CLP. Previous studies have shown that 2CLP results in pulmonary changes consistent with ARDS (14). These alterations include neutrophil accumulation, septal thickening, and hyaline membrane formation (see Figure 3, top row). To determine whether the previously demonstrated failure of HSP-70 expression (5) is in part responsible for the development of these abnormalities, we examined lung tissue after treatment with AdHSP. Representative sections of hematoxylin and eosin-stained lung tissue are depicted in Figure 3. Sections were examined 48 hours after viral administration and either no operation or 2CLP. At the same timepoint, there were no changes in unoperated animals administered AdHSP (data not shown). In animals given AdGFP at the time of 2CLP, we observed changes characteristic of ARDS and consistent with previous studies. These include neutrophil infiltration and septal thickening, as well as protein and fluid accumulation in the interstitial and alveolar spaces. However, these changes were attenuated dramatically in rats given AdHSP. Low-power sections $(\times 5$ and $\times 10)$ demonstrate the heterogeneous nature of ARDS following AdGFP and the near-normal appearance after administration of AdHSP. Higher resolution $(\times 20$ and $\times 40)$

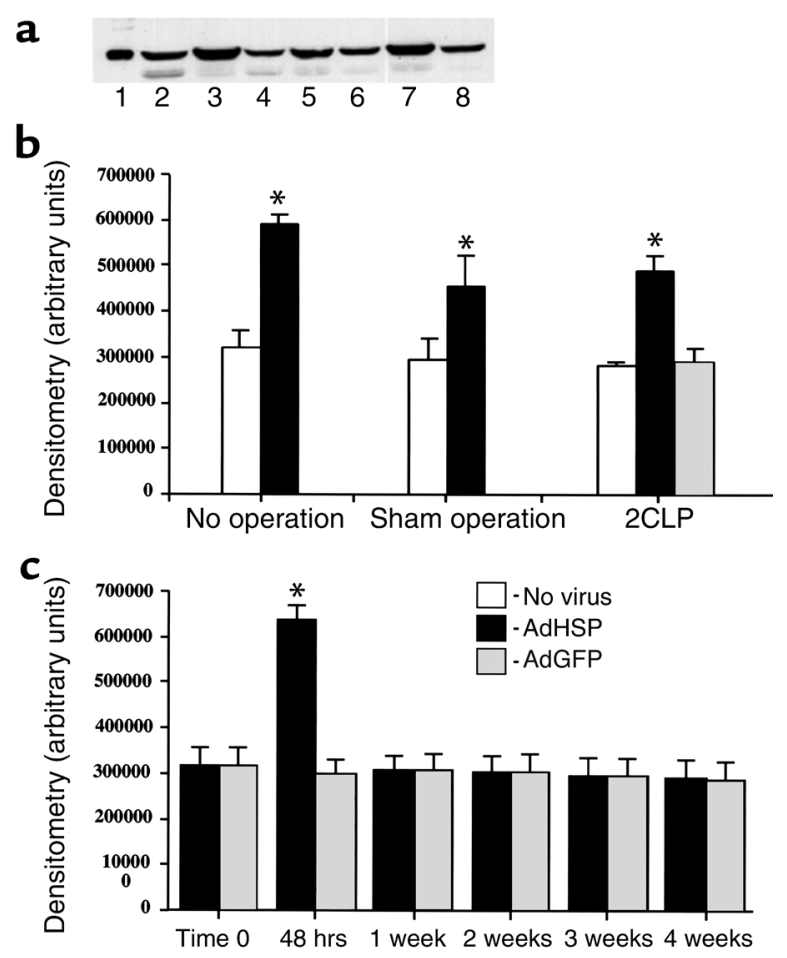

Figure 2

Immunoreactive HSP-70 levels in lung homogenate. (a) Representative immunoblot. Homogenate was obtained 48 hours after virus administration and operative intervention. Ten micrograms of homogenate were loaded per lane. Primary antibody was polyclonal goat anti-rat HSP-70; secondary antibody was rabbit antigoat IgG. Detection was with enhanced chemiluminescence. Lane 1, purified HSP-70 (positive control); lane 2, unoperated control without AdHSP; lane 3, unoperated control with AdHSP, lane 4, sham operation without AdHSP; lane 5, sham operation with AdHSP; lane 6, 2CLP without AdHSP; lane 7, 2CLP with AdHSP; lane 8, 2CLP with AdGFP. (b) Graphic representation of densitometric quantification of HSP-70 immunoblots. $n=3$ for each intervention. ${ }^{*}$ Significantly different $(P<0.05)$ from value without virus administration. Values are arbitrary densitometric units, expressed as mean \pm SD. (c) Graphic representation of densitometric quantification of AdHSP and AdGFP expression over time following sham operation. Values are mean \pm SD of densitometric measurements made on three immunoblots. ${ }^{*}$ Significantly different $(P<0.05)$ from all other measurements. 
Table 1

AdHSP expression over time following sham operation

\begin{tabular}{lccc}
\cline { 2 - 4 } Time & With AdHSP & Without AdHSP & With AdGFP \\
0 & $330,128 \pm 6,378$ & $333,214 \pm 9,391$ & \\
48 hours & $684,075 \pm 7,067^{A}$ & $334,931 \pm 5,595$ & \\
1 week & $314,441 \pm 9,258$ & $321,264 \pm 9,477$ & \\
2 weeks & $328,589 \pm 9,651$ & $318,506 \pm 8,389$ & \\
3 weeks & $309,077 \pm 7,591$ & $340,113 \pm 7,092$ & \\
4 weeks & $309,129 \pm 7,067$ & $330,146 \pm 6,391$ & $305,493 \pm 7,771$
\end{tabular}

Data are expressed as arbitrary densitometric units (mean \pm SD) from autoradiographs of Western blots at the indicated times after sham oper-

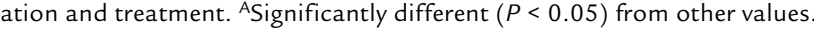
$n=2$ at each timepoint.

demonstrates the lack of consolidation, decreased cellularity, reduced septal edema, and absence of proteinaceous exudate that accompanied AdHSP instillation. Neutrophil counts further substantiate the effect of AdHSP on inflammation (Table 2). These data indicate the presence of few neutrophils in unoperated animals with or without virus, in sham-operated animals with or without virus, and in 2CLP animals treated with AdHSP. In contrast, 48 hours after 2CLP, with or without AdGFP administration, there was a dramatic increase in neutrophil accumulation. Thus, AdHSP administration significantly reduced pulmonary inflammation following 2CLP.

AdHSP decreases 48-hour mortality after 2CLP. Table 3 depicts mortality from 2CLP 48 hours after administration of either AdGFP or AdHSP. In our previous studies, approximately $25 \%$ of untreated animals subjected to 2CLP survived to 48 hours. Of 94 rats administered AdGFP in our current study, 65 (69\%) had died by 48 hours. In animals given AdHSP, only 32 of 84 (38\%) had succumbed. By $\chi$-square analysis, this was significant at $P<0.001$.

\section{Discussion}

Our data demonstrate that HSP-70 expression in lung can be increased using AdHSP, an adenovirus carrying the gene for HSP-70. Our data support the hypothesis that treatment with AdHSP attenuates neutrophil accumulation, septal thickening, interstitial fluid accumulation, and alveolar protein exudate 48 hours after 2CLP. These changes are characteristic of ARDS. Furthermore, AdHSP administration significantly decreases 48-hour mortality. These findings have important ramifications with regard to the pathogenesis and treatment of ARDS. In addition, our results may indicate an important, novel application for adenovirus-mediated gene therapy.

Importantly, heat pretreatment and/or HSP-70 elaboration has been shown to protect cells, reduce inflammation, and alter activation of transcriptional pathways both in vivo and in vitro (15-30). The disruption of normal cellular function that occurs in ARDS is just the sort of stimulus that ought to provoke a heat shock response. Nevertheless, our previous studies indicate that HSP-70 elaboration is not increased after 2CLP (5).

The heat shock response represents a classic, phylogenetically conserved mechanism of cellular protection (15) that has evolved to protect cells from untoward environmental perturbations. Activation of this pathway by any of a number of noxious stimuli - heat, hypoxia, hypoglycemia, transition metal intoxication, ischemia/reperfusion, endotoxemia, or shock - results in the elaboration of a series of heat shock proteins with specific cytoprotective activity (16-20). Of these, the most widely studied is the $70-\mathrm{kDa}$ HSP-70. Stressinduced increases in the expression of HSP-70 have been demonstrated in a number of tissues, including lung, kidney, heart, and liver (15-20). Of these tissues, the lung is unique in that there is HSP-70 expression in the absence of insult (15). This likely reflects continuous exposure to the environment.

Previous studies document that 2CLP fails to enhance HSP-70 in the lung (5). These findings are consistent with the effects of sepsis on HSP-70 expression in organs other than the lung $(12,13)$, and are also in keeping with our demonstration of a hepatic transcriptional defect in sepsis $(8,9)$. In all cases, this response is pathologic. Conversely, there are data indicating that heat pretreatment before a variety of insults protects cells, inhibits proinflammatory

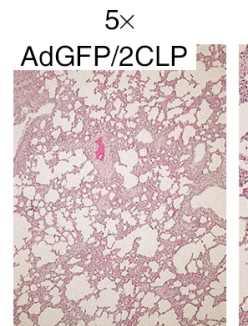

$5 \times$

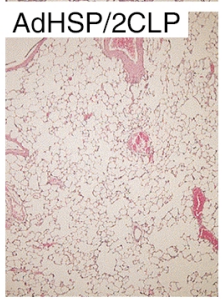

AdGFP/No op

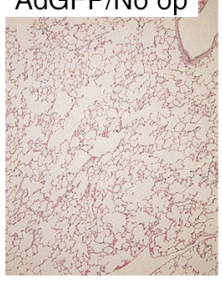

Figure 3

Sections from lungs harvested 48 hours after virus administration with or without 2CLP, stained with hematoxylin and eosin. Magnifications are indicated at top of figure. No op, no operation. 
Table 2

Neutrophil counts/high-powered field in fixed lung parenchyma

\begin{tabular}{lc}
\hline Intervention & Neutrophils \\
No operation & $12 \pm 4$ \\
No operation + AdGFP & $8 \pm 2$ \\
Sham operation & $17 \pm 6$ \\
Sham operation \pm AdGFP & $18 \pm 5$ \\
2 CLP & $914 \pm 156^{\mathrm{A}}$ \\
$2 \mathrm{CLP}+$ AdGFP & $877 \pm 144^{\mathrm{A}}$ \\
$2 \mathrm{CLP}+$ AdHSP & $21 \pm 9$
\end{tabular}

Data expressed as arbitrary units, mean \pm SD. ASignificantly different $(P<0.05)$ from other values.

cytokine induction, alters activation of transcriptional pathways, and prevents apoptosis both in vivo and in vitro (15-33). Indeed, studies have demonstrated that heat treatment significantly improves the outcome of phospholipid A1-mediated acute lung injury or systemically induced ARDS $(21,22)$. This inspired our current approach of enhancement of an impaired heat shock response using an adenovirus. Our data are unique; in other studies on sepsis and ARDS, the entire heat shock response, comprising a series of peptides, is invoked throughout the entire organism. In contrast, in the studies presented here, we have attempted to increase only HSP-70, a single peptide, in one organ, the lung.

Three potential mechanisms to explain the protective effects of HSP-70 have been investigated: preservation of protein structure and configuration $(15,34$, $35)$, attenuation of cytokine-induced inflammation $(15,29,36)$, and blockade of apoptosis $(15,30)$. In the studies detailed here, we found a reduction in the infiltration of neutrophils into lung parenchyma. Our studies were not designed to examine protein preservation or altered cell death. Indeed, attempts to quantify the effects of AdHSP on preservation of pulmonary epithelial cells failed. As a result of the enormous increase in inflammatory cells that accompanied 2CLP, the change in interstitial water/protein content, and an inability to distinguish epithelial cells from endothelial cells, it became impossible to count epithelial cells, even using fluorescent markers. However, damage to proteins and loss of cells are known components of ARDS (3). Given the improved histology and decreased mortality that accompanied AdHSP administration, it is likely that both processes were attenuated by treatment. Ongoing studies will address the effects of AdHSP on the structure and function of specific intrapulmonic proteins.

The AdHSP-associated reduction in mortality after 2 CLP came as a surprise. However, this study demonstrated a reduction in mortality only within the first 48 hours. We have no data on how AdHSP affects longterm mortality. Studies in human ARDS, especially in response to injury remote from the lung, indicate that pulmonary dysfunction is a rare cause of death (37).
Furthermore, as result of improved supportive care (ventilatory management, appropriate fluid administration, judicious use of antibiotics, and a host of other treatments), early mortality has become increasingly unusual. Rather, patients succumb, usually several weeks after the inciting event, to multiple organ dysfunction syndrome. Abnormalities are evident in a number of organ systems (lung, liver, kidney, heart, vasculature, and central nervous system), but the cause of death is obscure. Indeed, most patients die when lifesustaining support is deemed futile and is removed. It is likely that the high mortality following 2CLP and the ability of intrapulmonic AdHSP to attenuate this process results from the absence of life support modalities in this model. It is possible that intrapulmonic administration of AdHSP alters responses in organs other than the lung. In a previous study, we did not detect GFP in heart, liver, or kidney following intratracheal administration of AdGFP at the time of 2CLP (14). Therefore, altered mortality as a result of increased HSP-70 expression in tissues other than lung is unlikely. Furthermore, extension of the observation time to a period when cardiac dysfunction, renal insufficiency, or coagulopathy become evident might eliminate the putative protection afforded by intrapulmonary AdHSP.

Our previous study indicated that adenoviral uptake following 2CLP occurred primarily in pulmonary epithelial cells, especially type II pneumocytes (14). However, we did observe some uptake by pulmonary macrophages. AdHSP could well affect the behavior of these cells, reducing neutrophil accumulation via an alteration in chemokine expression.

We chose to examine long-term expression after sham operation for two reasons. Long-term survival from 2CLP, even after AdHSP administration, has not been demonstrated. Survival of sham operation, however, is virtually assured. In contrast to results in unoperated controls, viral uptake and expression after sham operation is robust (14). Hence, sham operation represents a logical intermediate approach.

The findings presented here have important implications with regard to future applications of gene therapy. Previous studies have focused on correcting inborn errors and permanent abnormalities in the genome. A major obstacle to the use of gene therapy in these disorders is that adenoviral DNA does not integrate into the chromosomes of the host cells. Therefore the response lasts for no more than several months (37). In ARDS, altered HSP-70 expression is required for only a short period of time. Indeed, long-term expression may be harmful.

\section{Table 3}

48-hour mortality after 2CLP followed by treatment with AdHSP or AdGFP

\begin{tabular}{ll}
\hline CLP + AdHSP & 2 CLP + AdGFP \\
$32 / 84(38 \%)$ & $65 / 94(69 \%)$
\end{tabular}

Shown are no. deaths/total no. animals undergoing treatment. 
Therefore, our demonstration that AdHSP expression after sham operation lasts no longer than 1 week is particularly important.

Finally, we administered AdHSP at the time of 2CLP. Since viral uptake and gene expression are not immediate, HSP-70 expression developed concurrently with the disease process. This avoids some of the concerns that accompany pretreatment strategies. Indeed, it is likely that the time course mimics that which would accompany an endogenous heat shock response. Our previous work indicates that cells are most receptive to viral uptake and transgene expression in the presence of inflammation (14). However, this approach does not eliminate the need to identify patients at risk. Therefore, future studies will focus on the timing of AdHSP administration.

In summary, we have demonstrated that administration of AdHSP reduces inflammation and improves short-term outcome in experimental ARDS. Furthermore, we have documented the efficacy of a new and exciting approach to gene therapy, transient expression of key intracellular proteins during an acute disease process. Future studies will focus on issues raised above and on identifying the mechanisms involved in the protective effect of HSP-70.

\section{Acknowledgments}

This work was supported by the Israeli Ministry of Health (General Scientist grant 4836) and by NIH grant R01 GM-59930.

1. Baue, A.E., Durham, R., and Faist, E. 1998. Systemic inflammatory response syndrome (MODS), multiple organ dysfunction syndrome (MODS), multiple organ failure (MOF): are we winning the battle? Shock. 10:79-89.

2. Milberg, J.A., Davis, D.R., Steinberg, K.P., and Hudson, L.D. 1995 Improved survival of patients with acute respiratory distress syndrome (ARDS): 1983-1993. JAMA. 273:306-309.

3. Ware, L.B., and Matthay, M.A. 2000. The acute respiratory distress syndrome. N. Engl. J. Med. 342:1334-1349.

4. The Acute Respiratory Distress Syndrome Network. 2000. Ventilation with lower tidal volumes as compared with traditional tidal volumes for acute lung injury and the acute respiratory distress syndrome. N. Engl. J. Med. 342:1301-1308.

5. Weiss, Y.G., Bouwman, A., Gehan, B., Raj, N., and Deutschman, C.S. 2000. Cecal ligation and double puncture impairs heat shock protein 70 (HSP-70) expression in the lungs of rats. Shock. 13:19-23.

6. Schears, G., Zheng, J., and Deutschman, C. 1999. Decreased transcription of surfactant proteins in an animal model of the adult respiratory distress syndrome (ARDS). Shock. 11(Suppl.):21. (Abstr.)

7. Andrejko, K.M., Chen, J., and Deutschman, C.S. 1998. Intrahepatic STAT-3 activation and acute phase gene expression predict outcome after CLP sepsis in the rat. Am.J. Physiol. 275:G1423-G1429.

8. Deutschman, C.S., Demaio, A., Buchman, T.G., and Clemens, M.G. 1993. Sepsis-induced alterations in phosphoenolpyruvate carboxykinase expression: the role of insulin and glucagon. Circ. Shock. 40:295-302.

9. Deutschman, C.S., et al. 1997. Sepsis-induced depression of rat glucose6-phosphatase gene expression and activity. Am. J. Physiol. 273:R1709-R1718.

10. Deutschman, C.S., et al. 1996. Increased expression of cytokine-induced neutrophil chemoattractant in septic rat liver. Am. J. Physiol. 271:R593-R600.

11. Goldman, M.J., and Wilson, J.M. 1995. Expression of alpha v beta 5 integrin is necessary for efficient adenovirus-mediated gene transfer in the human airway. J. Virol. 69:5951-5958.
12. Schroeder, S., et al. 1999. Impaired inducibility of heat shock protein 70 in peripheral blood lymphocytes of patients with severe sepsis. Crit. Care Med. 27:1080-1084.

13. Ofensterin, J.P., Heidemann, S., Juett-Wilstermann, A., and Sarnaik, A. 1998. Endotoxin inhibits heat induced HSP-70 in rats. Crit. Care Med. 26(Suppl. 1):A138. (Abstr.)

14. Weiss, Y.G., et al. 2001. Adenoviral vector transfection into the pulmonary epithelium after cecal ligation and puncture (CLP) in rats. Anesthesiology. 95:974-982.

15. De Maio, A. 1999. Heat shock proteins: facts, thoughts, and dreams. Shock. 11:1-12.

16. Bellmann, K., et al. 1995. Heat shock induces resistance in rat pancreatic islet cells against nitric oxide, oxygen radicals and streptozotocin toxicity in vitro. J. Clin. Invest. 95:2840-2845.

17. Kloterhalfen, B., et al. 1997. The influence of heat shock protein 70 induction on hemodynamic variables in a porcine model of recurrent endotoxemia. Shock. 7:358-363.

18. Marber, M.S., Mestril, R., Chi, S.H., and Sayen, M.R. 1995. Overexpression of the rat inducible $70-\mathrm{kD}$ stress protein in a transgenic mouse increases the resistance of the heart to ischemic injury. J. Clin. Invest. 95:1446-1456.

19. Tacchini, L., Schiaffonati, L., Pappalardo, C., Gatti, S., and BernelliZazzera, A. 1993. Expression of HSP 70, immediate-early response and heme oxygenase genes in ischemic-reperfused rat liver. Lab. Invest. 68:465-471.

20. Wong, H.R., and Wispe, J.R. 1997. The stress response and the lung. Am. J. Physiol. 17:L1-L9.

21. Villar, J., et al. 1994. Induction of the heat shock response reduces mortality rate and organ damage in a sepsis-induced acute lung injury model. Crit. Care Med. 22:914-922.

22. Malloy, J., et al. 1997. Alterations of the endogenous surfactant system in septic adult rats. Am. J. Respir. Crit. Care Med. 156:617-623.

23. Jaatela, M., Wissing, D., Bauer, P.A., and Li, G.C. 1992. Major heat shock protein hsp70 protects tumor cells from tumor necrosis factor cytotoxicity. EMBO J. 11:3507-3512.

24. Wong, R.H., Ryan, M., Menendez, I.Y., Denenberg, A., and Wispe, J.R. 1997. Heat shock protein induction protects human respiratory epithelium against nitric oxide-mediated cytotoxicity. Shock. 8:213-218.

25. Wong, H.R., Menendez, I.Y., Ryan, M.A., Denedberg, A.G., and Wispe, J.R. 1998. Increased expression of heat shock protein-70 protects A549 cells against hyperoxia. Am. J. Physiol. 275:L836-L841.

26. Mestril, R., Giordano, F.J., Conde, A.G., and Dillmann, W.H. 1996. Adenovirus-mediated gene transfer of a heat shock protein 70 (hsp 70i) protects against simulated ischemia. J. Mol. Cell. Cardiol. 28:2351-2358.

27. Suzuki, K., et al. 1997. In vivo gene transfection with heat shock protein 70 enhances myocardial tolerance to ischemia-reperfusion injury in rat. J. Clin. Invest. 99:1645-1650.

28. Hiratsuka, M., Mora, B.N., Yano, M., Mohanakumar, T., and Patterson, G.A. 1999. Gene transfer of heat shock protein 70 protects lung grafts from ischemia-reperfusion injury. Ann. Thorac. Surg. 67:1421-1427.

29. Yoo, G.C., et al. 2000. Anti-inflammatory effect of heat shock protein induction is related to stabilization of I kappa B alpha through preventing I kappa B kinase activation in respiratory epithelial cells. J. Immunol. 164:5416-5423.

30. Mosser, D.D., et al. 1997. Role of the human heat shock protein HSP 70 in protection against stress-induced apoptosis. Mol. Cell. Biol. 17:5317-5327.

31. Rosenfeld, M.A., et al. 1992. In vivo transfer of the human cystic fibrosis transmembrane conductance regulator gene to the airway epithelium. Cell. 68:143-155.

32. Dong, J.Y., et al. 1996. Systematic analysis of repeated gene delivery into animal lungs with a recombinant adenovirus vector. Hum. Gene Ther. 7:319-331.

33. Helmbrecht, K., and Rensing, L. 1999. Different constitutive heat shock protein 70 expression during proliferation and differentiation of rat C6 glioma cells. Neurochem. Res. 24:1293-1299.

34. Laufen, T., et al. 1999. Mechanism of regulation of HSP-70 chaperones by DnaJ cochaperones. Proc. Natl. Acad. Sci. USA. 96:5452-5457.

35. Diamant, S., Peres-Ben-Zvi, A., Bukau, B., and Goloubinoff, P. 2000. Sizedependent disaggregation of stable protein aggregates by the DnaK chaperone machinery. J. Biol. Chem. 275:21107-21113.

36. Gabai, V.L., et al. 1997. HSP 70 prevents activation of stress kinases. A novel pathway of cellular thermotolerance. J. Biol. Chem. 272:18033-18037.

37. Montgomery, A.B., Stager, M.A., and Carrico, C.J. 1985. Causes of mortality in patients with the adult respiratory distress syndrome. Am. Rev. Respir. Dis. 132:485-489. 